\title{
Spieltheoretische Ansätze in der Öffentlichen Wirtschaft
}

\author{
Anreizkompatibel; Price caps; Regulierung; Spieltheorie; Strombörsen
}

Dieser Aufsatz stellt einige Ansätze der Spieltheorie vor, die für die öffentliche Wirtschaft von Bedeutung sind. In einigen Fällen liefert die öffentliche Wirtschaft sogar das Standardbeispiel, etwa die Strommärkte für die Konkurrenz in Angebotsfunktionen. Im Detail werden hier unterschiedliche spieltheoretische Konzepte (Matrixspiele, Bayessche Mechanismen, sequenzielle Spiele) auf Strombörsen, zur Gestaltung von Anreizen und auf Preisregulierung angewandt. ${ }^{1}$

\section{Einleitung}

Dieser Aufsatz versucht spieltheoretische Konzepte vorzustellen, die von Relevanz für die öffentliche Wirtschaft sind. Dabei ist an eine vollständige Darstellung nicht zu denken, weder für die Spieltheorie ${ }^{2}$ (für dieses Gebiet gibt es hervorragende Fachbücher sowohl von einfachen für Manager zugeschnittenen Werken ${ }^{3}$ bis hin zu umfassenden und technisch anspruchsvolleren Referenzen ${ }^{4}$ ) noch für Anwendungen im öffentlichen Bereich. In der Tat, und dies ist die Motivation für diesen Aufsatz, ist vielen Praktikern dieser spieltheoretische Gehalt in zahlreichen dieser Anwendungen oft nicht bewusst, aber auch umgekehrt Theoretiker übersehen Entsprechungen ihrer Modelle im Energiemarkt. Zum Beispiel hat das von Klemperer-Meyer ${ }^{5}$ analysierte und über Konsulentenbeispiele motivierte Konkurrenz in Angebotsfunktionen seine reale Entsprechung in den Strombörsen, etwa dem englischen Pool gefunden. ${ }^{6}$ Dieses Beispiel der Strombörsen ${ }^{7}$ wird im folgenden Kapitel aufgegriffen. Aspekte asymmetrischer Information und strategischer Handlungen bestimmen das Design von Auktionen (z.B. für Frequenzen, die vormals, oft kostenlos vergeben wurden), öffentliche Ausschreibungen, die Gouvernanz öffentlicher

1 Der Autor dankt Michael Stadler und Hans Auer für Diskussionen zum Thema Strombörsen.

2 Cournot, Bertrand und auch Borel sind ohne Zweifel Vorläufer der Spieltheorie, die mit dem bahnbrechenden Werk von John von Neumann und Oskar Morgenstern „Theory of Games and Economic Behavior“ 1944 dann endgültig das Licht der Welt erblickt. Die weiteren wichtigen Stationen wurden dann mit dem Nobelpreis 1998 gewürdigt: John Nash für das Nash-Gleichgewicht (eine Verallgemeinerung des bereits von Cournot und Bertrand verwendeten Gleichgewichts) und andere Arbeiten (axiomatische Fassung einer kooperativen Lösung, wie generell die Spieltheorie in kooperative und nichtkooperative zerfällt), Reinhard Selten für die Teilspielperfektheit (bei sequenziellen Spielen sind nicht alle Nash-Gleichgewichte relevant sondern nur jene ohne leere Drohungen) und Harsanyi für Spiele mit unvollständiger Information.

3 Vgl. McMillan (1992), Dixit/Avinash/Barry (1993), Dixit/Avinash/Skeath (1999), Ghemawat (1997).

4 Z.B. Fudenberg/Tirole (1992), Rasmusen (2001).

5 Klemperer/Meyer (1989).

6 Vgl. Green/Newbery (1992).

7 Vgl. Kreis (2004). 
Unternehmen, ${ }^{8}$ und das in der Elektrizitätswirtschaft so aktuelle (und auch angefeindete) „Benchmarking“.9

Der Zweck dieses Aufsatzes ist an für die öffentliche Wirtschaft relevanten Beispielen auf die strategischen und damit spieltheoretischen Aspekte hinzuweisen, allerdings ohne Anspruch auf Vollständigkeit. Trotzdem ist es angebracht, gleich zu Beginn zwei Begriffe - ein Spiel und sein Gleichgewicht - vorzustellen. Ein Spiel beschreibt eine Situation in der jeder der Beteiligten - die „Spieler“ $\{i=1, \ldots, n\}$ - aus einer Menge der zur Verfügung stehenden Strategien $S_{i}$ (endlich, wie in dem folgenden Beispiel, oder auch unendlich) auswählt, etwa $s_{i}^{j} \in S_{i}$, wobei die gewählten Strategien aller dann die Auszahlungen (Gewinne, Verluste) $v_{i}$ an die einzelnen Spieler bestimmen, ${ }^{10}$

$v_{i}=V_{i}\left(s_{1}^{j_{1}}, s_{2}^{j_{2}}, \ldots, s_{i}^{j_{i}}, \ldots, s_{n}^{j_{n}}\right), s_{k}^{j_{k}} \in S_{k}$.

Der zweite wichtige Begriff (nicht nur in den Wirtschaftswissenschaften sondern auch in der Biologie, siehe dazu den Pionier Maynard Smith ${ }^{11}$ ) ist der des Gleichgewichts, genauer eines Nash-Gleichgewichts $\left(s^{N}\right)$, das dann gegeben ist, wenn keiner der Beteiligten mehr einen Nutzen aus einer Änderung seiner Strategie ziehen kann, gegeben die Strategien all der anderen (d.h. alle folgenden Anwendungen sind nicht-kooperative Spiele, in kooperativen Spielen geht es primär um den Aufteilungsmodus). In anderen Worten, im Nash-Gleichgewicht ist die Strategie eines jeden Spielers die beste Antwort auf die Strategien der anderen Spieler, formal: ${ }^{12}$

$s_{i}^{N}=\underset{s_{i}^{j} \in S_{i}}{\arg \max } V_{i}\left(s_{1}^{N}, s_{2}^{N}, \ldots, s_{i-1}^{N}, s_{i}^{j}, s_{i+1}^{N}, \ldots, s_{n}^{N}\right)$ für alle $i$.

Dieser Ansatz, von Cournot und Bertrand bereits für das Duopol angewandt, bedeutet, dass jeder Spieler seine Strategie als Optimierungsproblem mit der eigenen Strategie als Instrument konditional auf die Strategien der anderen Spieler bestimmt im Wissen, dass die anderen dasselbe Kalkül durchführen (vgl. dazu die bekannten Überlegungen von Sherlock Holmes in seiner Verfolgung von Professor Moriarty in dem er sich in dessen Logik versetzt). Diese Annahme, dass sich die Interaktionen im Kopf abspielen und somit ohne Anpassung das Gleichgewicht resultiert, unterscheidet die Spieltheorie in weiten Teilen der Wirtschaftswissenschaften von der Biologie, wo dann Fragen der Stabilität und der Resistenz gegenüber Mutanten eine große Rolle spielen (evolutionär stabile Gleichgewichte).

8 Siehe Laffont/Tirole (1993).

9 Shleifer (1985).

10 Eventuell inklusive stochastischer Elemente, die allerdings in dieser Darstellung ausgespart bleiben.

11 Etwa Maynard (1982).

12 Im Falle kontinuierlicher Entscheidungen und innerer Lösungen bestimmt sich ein Nash-Gleichgewicht als die Lösung von den n-Gleichungen resultierend aus den üblichen Optimalitätsbedingungen erster Ordnung: $\frac{\partial V_{i}\left(s_{1}, \ldots, s_{i-1}, s_{i}, s_{i+1}, \ldots, s_{n}\right)}{\partial s_{i}}=0, i=1, \ldots, n$ 


\section{Strommärkte}

Strommärkte wie etwa der NordPool als auch der vormalige und viel beschriebene Pool in England und Wales sind durch eine so genannte Konkurrenz in Angebotsfunktionen charakterisiert. Das heißt, die Unternehmen geben Preisgebote für jede ihrer Produktionseinheiten ab. Aus solchen Geboten werden jene Anlagen aufgerufen, die zu jedem Zeitpunkt die (erwartete) anfallende Nachfrage zu den geringsten Kosten bedienen. Da sich die am Pool teilnehmenden Unternehmen über Preisgebote konkurrieren, so war vermutlich eine der Ausgangsüberlegungen vom Chefdesigner der britischen Privatisierung (Littlechild), werden die Unternehmen Grenzkostengebote abgeben in Analogie zu Bertrands Preiskonkurrenz im Duopol (als Antwort auf Cournots Ansatz, wo sich die Unternehmen in Mengen konkurrenzieren). Abbildung 1 zeigt eine solche für den Nordpool konstruierte Grenzkostenkurve. Trotzdem kam und kommt es in Strombörsen zu Preisspitzen am bekanntesten jene in Kalifornien im Jahr 2000, aber auch im NordPool, in Deutschland ${ }^{13}$ im Dezember 2001 und bereits wenn auch in geringerem Ausmaß im britischen Pool. Solches Verhalten soll nun mittels einfacher spieltheoretischer Ansätze untersucht werden.

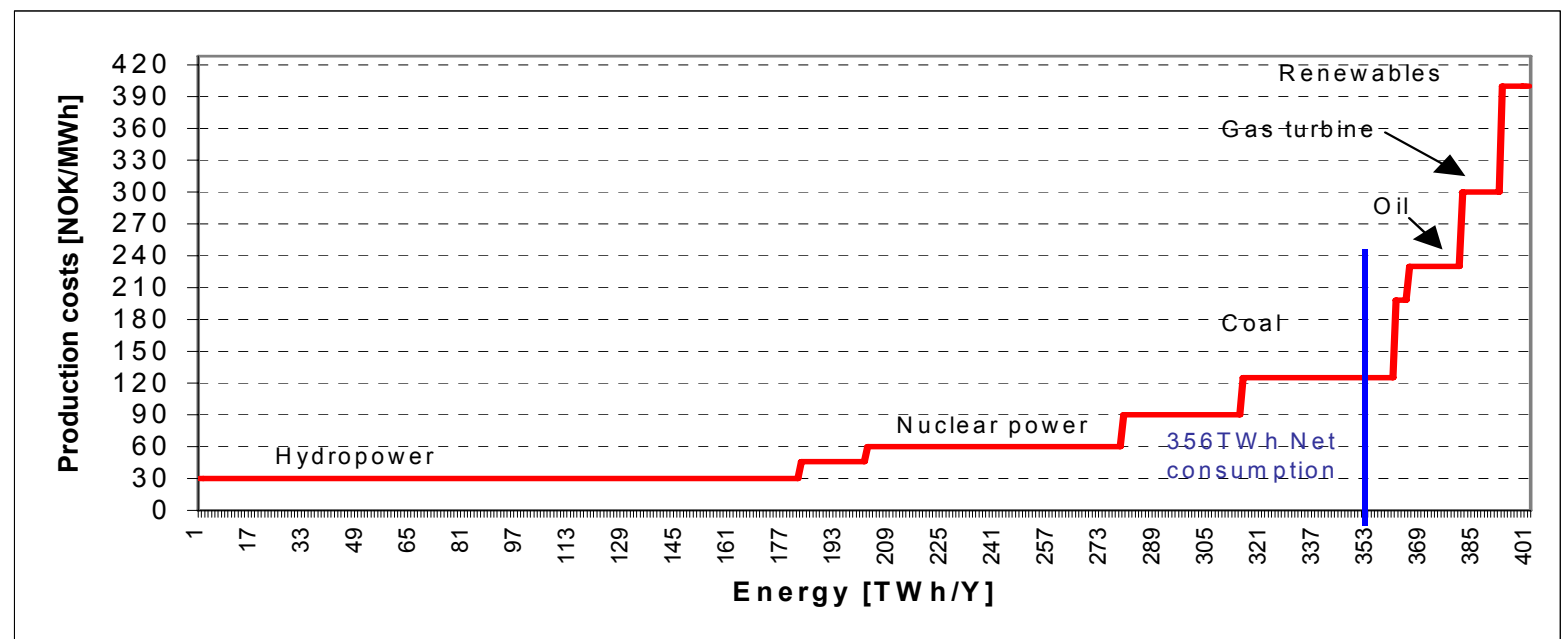

Abb. 1: Elektrizitätserzeugung im Nord Pool für 2000 (mit eingezeichneter Nachfrage), Nordpool ASA (2001), nach Typ aufsteigend gereiht nach den Kosten je kWh.

Quelle: Eigene Darstellung

Annahmen: Die aggregierte Nachfrage nach Elektrizität $D$ (eine gerade Zahl) wird von zwei Unternehmen ${ }^{14}$ gedeckt. Beide Anbieter verfügen über den gleichen Kraftwerkspark mit den gleichen Kosten, $\left\{c_{i}, i=1, \ldots K\right\}$. Jedes dieser Kraftwerke produziert eine Einheit, daher $K>1 / 2 D$ um die Nachfrage zu decken, und die Indizierung der Kraftwerke folgt der wirtschaftlichen Effizienz, $c_{i} \leq c_{j}$ für alle $i<j$. Um die strategischen Aspekte

13 Vgl. Leipzig Power Exchange, http://www.lpx.de, 2002.

14 Was dem britischen Pool entsprach, wo PowerGen und National Power über die marginalen Kraftwerke (und damit über den Preis) trotz weiterer Teilnehmer - Nuclear Power and zahlreiche kombinierte GasDampfturbinenproduzenten, vgl. Newbery (2002), entschieden. 
herauszuarbeiten, sei weiters angenommen, dass die Regulierungsbehörde bzw. der Auktionator die Kosten $c_{i}$ der einzelnen Kraftwerke kennt. Effizienter Einsatz verlangt, dass $n$ $=D / 2$ das marginale Kraftwerk ist, das den Preis mit $p=c_{n}$ festlegt. Dies liefert für jedes der Unternehmen den Gewinn

$$
\pi_{0}=c_{n} n-\sum_{i=1}^{n} c_{i}
$$

der Index 0 identifiziert diesen Referenzfall effizienten Kraftwerkseinsatzes.

Nach dieser Beschreibung des Rahmens, kommen wir zu den Strategien und ebenso wie die Anzahl der Spieler wird auch die Menge der Strategien auf zwei beschränkt:

$$
s_{i}^{j} \in S_{i}=\{\text { Kraftwerk } k<n: a=\text { bereitstellen, } b=\text { nicht bereitstellen }\}, i=1,2 .
$$

Durch Aussetzen (durch Vorgabe unerwarteter jedoch notwendiger Wartung) des an sich kosteneffizienten Kraftwerkes $k$ wird der Typ $n+1$ zum marginalen Kraftwerk, was den Preis auf $p=c_{n+1}$ erhöht. Klarerweise ist der Anreiz das teuerste Kraftwerk (also das $n$ te) aus dem Angebot zu streichen am größten, aber dies wäre zu offensichtlich, und deshalb sei angenommen, dass über Einsatz bzw. Nichteinsatz des effizienteren Kraftwerks $k$ entschieden wird. Im Falle, dass Spieler „1“ (durch den hochgestellten Index beschrieben) das Kraftwerk $k$ (auf Grund der Symmetrie gilt das gleiche auch für Spieler ,2“) nicht anbietet, ergibt sich folgender zusätzlicher Gewinn:

$\pi_{b}^{1}-\pi_{0}^{1}=\left(c_{n+1}-c_{n}\right) n-\left(c_{n+1}-c_{k}\right)$.

Diese Differenz ist positiv für hinreichend großes $n$, d.h. für eine große Nachfrage, jedoch negativ bei hohen Kostendifferenzen zwischen den Kraftwerken $j$ und $n$ and geringer Nachfrage. Der Gewinn des Spielers „2” bei gleichzeitigem Anbieten von $k$ ist:

$$
\pi_{a}^{2}-\pi_{0}^{2}=\left(c_{n+1}-c_{n}\right) n .
$$

Die Gewinne sind unabhängig davon, welcher der Anbieter die $(n+1)$ Einheit betreibt, weil diese ohnedies nur die Kosten deckt. Wenn beide anbieten, resultiert der effiziente Kraftwerkseinsatz und kein zusätzlicher Gewinn: $\pi_{a}^{1}-\pi_{0}=\pi_{a}^{2}-\pi_{0}=0$. Wenn beide nicht anbieten ergibt das

$$
\pi_{b}^{1}-\pi_{0}=\pi_{b}^{2}-\pi_{0}=\left(c_{n+1}-c_{n}\right) n-\left(c_{n+1}-c_{k}\right) .
$$

Und der letzte Fall, ,2“ bietet $k$ nicht an jedoch „1” schon, ist symmetrisch zum ersten Fall so, dass alle möglichen Auszahlungen zusammengefasst werden können, wobei die erste Zahl in den vier Zellen in Tabelle 1 sich immer auf den „Zeilenspieler“ „1“, und die zweite immer auf den Spaltenspieler „2“ bezieht. 
Tabelle 1: Zusatzgewinn $\mathrm{zu} \pi_{0}$ bei Bereitstellung/Zurückziehen des Kraftwerks $k$, $\Delta c=\left(c_{n+1}-c_{n}\right), \Delta c_{k}=\left(c_{n+1}-c_{k}\right)$,

$$
i=2
$$

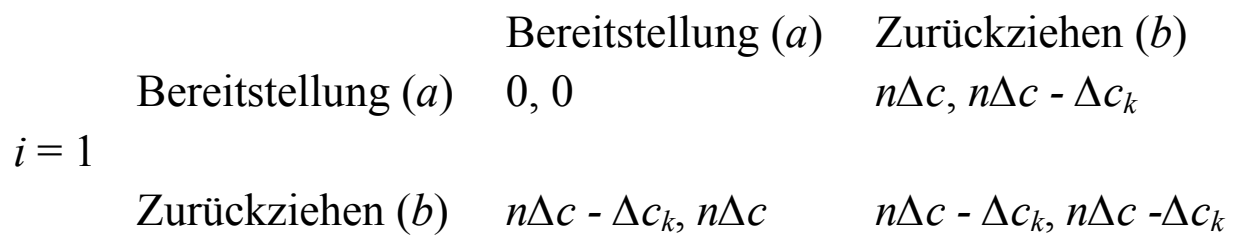

Fall 1: $n \Delta c-\Delta c_{k}<0$, d.h. niedrige Nachfrage oder große Kostendifferenz $\Delta c_{k}$. Dann ist das Nash-Gleichgewicht eindeutig und durch $(a, a)$, d.h. die Bereitstellung, und damit den effizienten Kraftwerkseinsatz, bestimmt. Dass dies ein Gleichgewicht ist, ist leicht nachzuprüfen, weil egal was der andere Spieler tut, die Strategie $a=$ Bereitstellen in keinem Fall zu einem schlechteren Ergebnis führt. Spieltheoretisch handelt es sich hier um das bekannte Gefangenendilemma was in diesem Fall (wie auch im Originalbeispiel) die Bezeichnung problematisch erscheinen lässt, weil dieses Dilemma zu effizientem Verhalten der Spieler (aus der Sicht der Konsumenten und der Gesellschaft) führt.

Fall 2: $\left(n \Delta c-\Delta c_{k}\right)>0$, d.h. hohe Nachfrage ( $n$ genügend groß) bzw. geringe Kostendifferenz (z.B. für $k=n$, das teuerste Kraftwerk kann aus dem Angebot genommen werden, reduziert sich diese kritische Differenz auf den immer positiven Ausdruck $(n-1) \Delta c$ $>0$ ). In diesem Fall ergeben sich aus der Anwendung von (2) gleich drei Nash-Gleichgewichte:

1. $(a, b)$, d.h. Spieler , $1{ }^{\prime \prime}$ bietet alle Kraftwerke inklusive dem $k$-ten an, während Spieler ,2“ das Kraftwerk $k$ aus seinem Angebot nimmt.

2. Auf Grund der Symmetrie des Spiels ist dann natürlich auch $(b, a)$ ein Gleichgewicht.

3. Die obigen beiden asymmetrischen Gleichgewichte beinhalten nur reine Strategien, $a$ oder $b$. Darüber hinaus gibt es ein weiteres und dann symmetrisches Gleichgewicht in so genannten gemischten Strategien. In diesem Gleichgewicht wird jede Strategie mit einer bestimmten Wahrscheinlichkeit, $\alpha$ für Strategie $a=$ Bereitstellen und $\beta=(1-\alpha)$ für $b=$ Zurückziehen, gespielt, wobei $\alpha=\Delta \mathrm{c}_{\mathrm{k}} /\left[\Delta \mathrm{c}_{\mathrm{k}}+(\mathrm{n}-1) \Delta \mathrm{c}\right]$,

d.h., die Wahrscheinlichkeit ${ }^{15}$ einer Bereitstellung (und damit effizienter Einsatz) steigt mit der Kostendifferenz und fällt in der Nachfrage. Diese im ersten Moment eher theoretisch erscheinende Lösung ist allerdings von höherer praktischer Relevanz, denn solche mit Zufallsfaktoren behaftete Strategien sind für den Regulator ungleich schwerer als Marktmanipulation zu erkennen als das Gleichgewicht in den reinen Strategien.

15 Die Wahrscheinlichkeiten werden dabei so bestimmt, dass der Gewinn der Spieler unabhängig von der Wahl der Strategie des anderen ist: $\Delta \pi \mathrm{a}=(1-\alpha) \mathrm{n} \Delta \mathrm{c}=\Delta \pi \mathrm{b}=\alpha(\mathrm{n}-1) \Delta \mathrm{c}+(1-\alpha)[\mathrm{n} \Delta \mathrm{c}-\Delta \mathrm{ck}]$. Einfaches Umformen liefert dann das Ergebnis. 
Dieser zweite Fall weist auf mehrere Gleichgewichte (gleich drei bei nur zwei Strategien), auf gemischte Strategien und auf das Koordinationsproblem (in Spielen mit mehreren Gleichgewichten) hin. Diese Problematik tritt in den verwandten Spielen „Game of Chicken ${ }^{16 ،}$ und „Kampf der Geschlechter ${ }^{17 ،}$ auf. Das Ergebnis mehrerer (oft sogar ,vieler") Gleichgewichte ist ein Charakteristikum strategischer Interaktion, das nicht als ein Schwachpunkt sondern eher als ein realistisches Ergebnis angesehen werden muss, was allerdings die Prognose- und damit Aussagefähigkeit gerade im Regulierungsumfeld einschränkt.

Dieses einfache Modell kann somit den Anreiz für Preisspitzen konditional auf die Nachfrage und auf die Effizienz der zurückzuziehenden Anlage herausarbeiten. Dies hat folgende auch empirisch testbare und für die Regulierungspraxis wichtige Implikationen: Es ist ungleich profitabler, teure als kosteneffiziente Kraftwerke zurückzuziehen, was die Wartungsintensität zugunsten teurer Kraftwerke verzerrt. Die Intensität „,technisch bedingter" Rücknahmen von Kraftwerken sollte außerhalb der Spitzenperioden ungleich seltener sein.

\section{Anreize}

Die Bedeutung von Anreizen im Wirtschaftsleben ist universell. Das Versagen des Sozialismus ist zu einem Großteil auf das Fehlen wirtschaftlicher Anreize zurückzuführen ${ }^{18}$. Die Reformen in der chinesischen Landwirtschaft zwischen 1978 und 1984 führten zu einer $60 \%$ igen Produktionssteigerung ${ }^{19}$, und wie anders als durch geänderte Anreize ist die wirtschaftliche Entwicklung der vorher jahrzehntelang stagnierenden Wirtschaft der Volksrepublik China zu erklären? Im Sozialbereich führen Anreize zu teilweise unfinanzierbaren Auswüchsen. So haben die Niederlande einerseits die höchste Lebenserwartung in Europa, andererseits mit 13\% der arbeitenden Bevölkerung die höchste Invalidenrate und zwar, weil die Niederlande eine großzügige Unterstützung gewährt. ${ }^{20}$ Die Situation ist in Österreich ähnlich, wo ein Drittel im Alter zwischen 55 und 64 Jahren Behindertenzulagen bezieht. In Harvard hat sich die Anzahl der Studenten verdoppelt, die um Hilfe und Sonderbehandlung auf Grund des Disabilities Act ansuchen, der auch Rechtschreibschwäche als Behinderung anerkennt und wo HIV-Infektion als nächstes auf die Liste kommt ${ }^{21}$, und das ist nur der Anfang, so haben auf der Universität Boston $40 \%$ der , lern-

16 Bekannt aus dem Film „Denn sie wissen nicht, was sie tun (A rebel without a cause)“ mit James Dean, wo zwei Jugendliche gegen den Abgrund rasen und der gewinnt, der später bremst.

17 Mit den Gleichgewichten in den reinen Strategien entlang der Hauptdiagonale, also (a, a) oder (b, b), weil gemeinsam verbrachte Abende, $a=$ beim Boxkampf (was der „Mann“ bevorzugt), b = Ballett (was die „Frau“ lieber sehen würde), beiderseits bevorzugt werden. Die Ähnlichkeit zum obigen Spiel (Fall 2) besteht darin, dass jeder Spieler eines der Gleichgewichte bevorzugt; darüber hinaus gibt es noch die gemischte Strategie einige Abende bei Boxkämpfen und die anderen bei Ballettvorführungen zu verbringen.

18 Selbst in seiner großen polito-ökonomische Kontroverse ließ Hayek in seiner Kritik am Planungssozialismus a la Lange und Lerner Anreize draußen, weil dies ein soziologisches Phänomen sei, siehe Levy (1990).

19 Vgl. McMillan/Whalley/Zhu (1989).

20 Bolkestein (1999) und auf S. 18, ,down with disablement“".

21 The Economist (April 18th, 1999) S 47. 
schwachen“ (,learning-disabled“), Studenten die High School absolviert, ohne dass ihr Problem auch nur diagnostiziert wurde. ${ }^{22}$

Die Bedeutung privater Information erkennt man auch daran, dass dafür mittlerweile zwei Nobelpreise an 5 Wissenschaftler (zuerst 1996 an Vickerey und Mirrlees, und dann 2001 an Akerlof, Spence und Stiglitz) vergeben wurden. Diese Ansätze beachten explizit die menschliche (aber auch bereits bei Primaten ausgeprägte) Tendenz, Dinge zu den eigenen Gunsten auszulegen und gelegentlich sogar zu manipulieren. ${ }^{23}$ Die Berücksichtigung von solch potenziell strategischem Verhalten scheint mir einer der Grundlagen der Managementwissenschaften ${ }^{24}$ und auch von großer Bedeutung für die öffentliche Wirtschaft zu sein, dem leider nicht immer Rechnung getragen wird. Zwar geschieht dies zunehmend zumindest auf internationaler Ebene (etwa bei den letzten Wirtschaftskrisen in Asien Lateinamerika, der Entschuldung der ärmsten Länder, möglicherweise inklusive sogar des OPEC Mitglieds Nigerien, und bei der Kritik am Internationalen Währungsfond wurden Begriffe wie (un-) moralisches Hassard verwendet), aber andererseits werden selbst nach Jahrzehnten der Informationsökonomik (bekannte) Konsequenzen gerade im öffentlich-wirtschaftlichen Bereich oft (bewusst?) ignoriert, wie die obigen Beispiele dokumentieren. Und dies obwohl die Notwendigkeit der so genannten Anreizkompatibilität auch vor dieser ökonomische Theorie bekannt war, wie die folgende Beschreibung aus Elias Canetti, Die Stimmen von Marrakesch, Fischer Taschenbuch (1996, Original 1954, S. 17) der Preisgestaltung in den Souks von Marrakesch deutlich zeigt: „Es gibt Preise für Arme und Preise für Reiche, wobei die für die Armen natürlich die höchsten sind." Bemerkenswert dabei ist nicht, dass die Händler wissen, was anreizkompatibel ist (denn die, die es nicht wissen, gehen schnell bankrott) sondern, dass der Literat (und promovierte Chemiker) Canetti dies so selbstverständlich findet, dass er dem keine Erklärung hinzufügt. Trotzdem sei hier eine Erklärung nachgeliefert, wobei Preisdifferenzierung als effiziente Händlerstrategie vorausgesetzt wird. ${ }^{25}$ Unter dieser Voraussetzung gilt bei vollständiger Information eine Preisgestaltung nach der (marginalen) Zahlungsbereitschaft, wobei die Reichen mehr zahlen würden. Jedoch bei privater Information (,reich“ oder ,arm“ zu sein) funktioniert dies nicht mehr, weil dann alle Käufer einen Anreiz haben, vorzugeben, arm zu sein (etwa im billigsten Jutekaftan einkaufen zu gehen). Dieses Kundenverhalten unterläuft die intendierte Preisdifferenzierung, weil durch dieses „Schwindeln“ nur die niedrigen Preise für die ,armen“ Kunden zur Anwendung kommen. Hingegen ist die von Canetti beobachtete Preisdifferenzierung anreizkompatibel, denn es zahlt sich kaum aus, einen Armani-Anzug zu erstehen, um in den Genuss der für Reiche günstigeren Preise zu kommen. Tatsächlich kann die Berücksichtigung privater Information (d.h. der Anreizkompatibilität) wirtschaftspolitische Empfehlungen gegenüber vollständiger Information umdrehen, z.B. beim Energiesparen. ${ }^{26}$

22 Allen (July 10th, 1996).

23 Hobbes, zitiert nach Frans B. M. de Waal (2005) S. 55.

24 C. Seidl „Aufgabe ... vornehmlich der Betriebswirtschaftslehre müsste sein, das menschliche Antlitz [des Unternehmers] in ihren Lehrgebäuden angemessen zu berücksichtigen“, in: Wagner (Hrsg.)

25 Mitsutishi (1999) zeigt, dass in kleinen Märkten solche Basarmethoden gegenüber Preisauszeichnung bestehen können.

26 Wirl (1999). 
Klassische Probleme bedingt durch private Information sind die Beziehungen zwischen Eigentümer und Manager, Kreditgeber und Kreditnehmer, Versicherung und Versicherungsnehmer, wobei entsprechend der Hierarchie der Entscheidungen von einem Prinzipal- (Eigentümer, Bank, Versicherung) und Agenten- (Manager, Kreditnehmer, Versicherter) -problem gesprochen wird. Das Beispiel von Versicherungen eignet sich gut, die Konsequenzen von privater Information darzustellen, wobei man zwischen adverser Selektion und moralischem Hassard unterscheidet. Betrachten wir das Beispiel einer Fahrradversicherung. Ignorieren von privater Information führt dazu, dass die Versicherung das durchschnittliche Diebstahlsrisiko kennt (etwa durch statistische Auswertungen) und dies zur Grundlage für die Prämie macht. Kann die Versicherung dann mit dieser Prämie die dann tatsächlich eintretenden Schadensfälle decken? Versicherungsmathematisch wäre die Antwort ja, trotzdem ist damit keine Deckung möglich, ja möglicherweise kann eine solche Versicherung überhaupt nicht kostendeckend offeriert werden. Der Grund ist, dass das individuelle Risiko eines Fahrraddiebstahls private Information ist. So hat ein potenzieller Versicherungsnehmer ein teures Rennrad, das er nur zum Fahren verwendet und sonst sicher in der Garage verstaut. Dieser Fahrradbesitzer hat de facto kein Diebstahlsrisiko, was in die Statistik des durchschnittlichen Risikos eingeht, und schließt deshalb keine Versicherung ab. Ein anderer Typ (ein häufig gebrauchter Begriff in dieser Literatur) verwendet sein Fahrrad als Transportmittel im Alltag und lässt es, wenn auch abgesperrt an Bahnhöfen und Plätzen stehen. Dieser Radfahrer erkennt, dass er ein überdurchschnittliches Risiko eines Diebstahls hat. Deshalb ist für ihn ein Versicherungsvertrag mit einer Prämie basierend auf dem mittleren Risiko profitabel. Dies hat zur Folge, dass die Versicherung überwiegend Verträge mit jenen Typen abschließt, die ein hohes Risiko darstellen aber kaum mit niedrigen Risiken. Dies nennt man adverse Selektion und diese kann soweit gehen, dass selbst bei Antizipation des erhöhten Risikos kein Gewinn für die Versicherung möglich ist, sodass auf Grund privater Informationen an sich gewünschte Versicherungen unterbleiben ${ }^{27}$ (nota bene eine Begründung für eine umfassende, bei uns öffentliche Krankenversicherungen, um diesen Sortierungseffekt zu verhindern). Wenn im obigen Beispiel sich auch noch das Verhalten ex post ändert, z.B. das Rad wird auf Grund der Versicherung nun auch an riskanteren Plätzen abgestellt, spricht man von moralischem Hassard, was die Situation der Versicherung weiter verschlechtert. Diese Möglichkeit, private Information strategisch für sich zu nützen, bringt das spieltheoretische Element ein, wobei es sich typischerweise um ein sogenanntes Bayessches Gleichgewicht in einem Spiel mit unvollständiger Information handelt, deren Analyse ursprünglich auf Harsanyi zurückgeht. Dazu wird das Beispiel eines einfachen Arbeitsvertrags im Anhang abgeleitet und dabei wird auf einige Konsequenzen hingewiesen.

Die Implikationen privater Information sind zwar den Händlern in den Souks, Elias Canetti und auch den Managern klar, aber werden in der öffentlichen Wirtschafts- und Sozialpolitik wie in den bereits erwähnten Beispielen (und in concretowurde bei der Einführung der Pflegeversicherung in Österreich schlicht und einfach „übersehen“, dass mit dieser Leistung, die Pflegefälle ansteigen werden) ignoriert. Auch im energiewirtschaftli-

27 Tatsächlich sind solche Versicherungen (Rad, Schi) selten, von kurzer Dauer, oder sehr stark eingeschränkt. 
chen Bereich wurde und wird die Anreizkompatibilität vernachlässigt, etwa in den zahlreichen Energiesparprogrammen ${ }^{28}$ und international bei Joint Implementation ${ }^{29}$ im Rahmen der Kyoto Klimakonvention.

\section{Sequenzielle Spiele, extensive Form und Verpflichtung}

Die bisher analysierten Spiele waren in Matrixform mit den Auszahlungen als Zelleinträgen gegeben. Obwohl sich viele Spiele auf diese Form reduzieren lassen, geht dabei oft Information über die tatsächlichen Entscheidungsabläufe- und -folgen verloren. Zur Demonstration dient im folgenden das Beispiel Regulierung: Der (politische) Entscheidungsträger kann zwischen Durchschnittskostenregulierung $(\phi)$ und Preisobergrenzen (Price Caps, $\bar{p}$ ) wählen. Das Unternehmen kann sich anstrengen (investieren) und damit die Effizienz steigern $(I)$ oder nicht $(0)$. Dies ergibt in der bisher üblichen Matrizenschreibweise (der sogenannten Normalform) folgende Auszahlungen:

\section{Unternehmen}

$\begin{array}{lll} & 0 & I \\ \phi & 0,0 & 2,-1\end{array}$

Regulierungsbehörde

$\bar{p} \quad-1,1 \quad 1,2$

\section{Tab. 3: Auszahlungen beim Regulierungsspiel}

Quelle: Eigene Darstellung

Die Zahlen kommen dabei so zustande: Der Payoff an die beiden Spieler bei Durchschnittskostenregulierung (Strategie , $\phi^{\prime)}$ ) und geringem Einsatz des Unternehmens (Strategie, $0^{\circ)}$ ) dient als Normierung und ist daher auf jeweils Null gesetzt. Wenn trotz Durchschnittspreisregulierung investiert wird, lukriert die Regulierungsbehörde (über niedrigere Preise) den Gewinn, während das Unternehmen für die Anstrengungen, Kosten zu senken, nicht kompensiert wird. Umgekehrt nützen Price Caps und keine Anstrengung dem Unternehmen, weil dem Unternehmen eine Marge zugestanden wird, wofür die öffentliche Hand aufkommt. Das effiziente Ergebnis von sowohl fixen Preisobergrenzen als auch Investitionen nützt beiden Parteien, allerdings mehr dem über einen Informationsvorsprung verfügenden Unternehmen, daher die Auszahlungen $(1,2)$; diese Asymmetrie ist nicht essentiell, denn dasselbe Gleichgewicht resultiert auch für $(1.5,1.5)$ in der südöstlichen Zelle. Dieses Spiel hat wie das Spiel in Tabelle 1 und das Gefangenendilemma ein Gleichgewicht in dominanten (und leider ineffizienten) Strategien: $(\phi, 0)$. Dies bedeutet, dass Regulierung hier unweigerlich in einer Blockade endet, die jedoch aufgelöst werden kann durch ein sequenzielles Spiel, wie es in dem Entscheidungsbaum

28 Wirl (1997).

29 Wirl/Huber/Walker (1998). 
in Abbildung 2 (dies nennt man die extensive Form des Spiels) dargestellt ist. In dieser Darstellung zieht die Regulierungsbehörde zuerst und dann erst zieht das Unternehmen. Da die Regulierungsbehörde weiss, dass im Teilspiel für die Strategie $(\phi)$ das Unternehmen 0 wählt, ${ }^{30}$ resultiert in dieser sequenziellen Abfolge dann das Gleichgewicht $(\bar{p}, I)$, was die Regulierungsblockade auflöst und nun ein effizientes Ergebnis für die öffentliche Regulierung mittels Preisobergrenzen liefert.

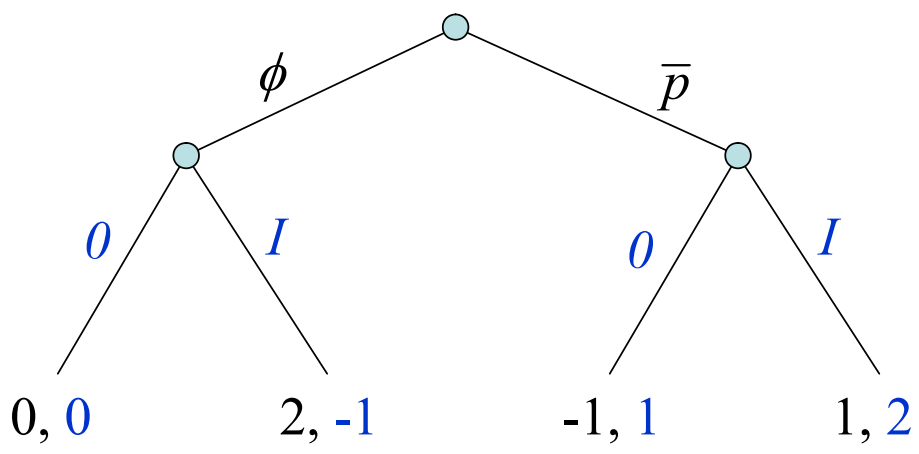

Abb. 2: Regulierung (Preisobergrenzen $\bar{p}$ versus Durchschnittstarife $\phi$ ) als sequenzielles Spiel (in extensiver Form) zwischen der Regulierungsbehörde und dem regulierten Unternehmen, das investieren kann (I) oder nicht (0)

Quelle: $\quad$ Eigene Darstellung

Aber Halt! Dieses sequenzielle Spiel unterstellt, dass sich die Regulierungsbehörde (glaubwürdig) zur Strategie $\bar{p}$ binden kann, egal was passiert, was eine leider unrealistische Annahme ${ }^{31}$ ist. Dieses Problem der mangelnden Glaubwürdigkeit von politischen Versprechen ist ein wichtiger für politische Rahmenbedingungen besonders zu beachtender Aspekt. Im konkreten Problem provozieren hohe Gewinne egal ob durch glückliche Umstände oder auf Grund enormer Anstrengungen politische Entrepreneurs (oder auch Boulevardblätter), diese Profite anzuprangern. Dazu kommt, dass die öffentliche Hand gleich mehrere Möglichkeiten zum Vertragsbruch hat, wie „freiwillige“ Nachverhandlung, neue Vorschreibung oder Aneignung der Gewinne durch eine nachträgliche Besteuerung, wie es bspw. die englische Labour-Regierung nach der Regierungsübernahme 1997 machte, als sie eine Steuer auf windfall gains der Elektrizitätsindustrie im Ausmaß von £2,4 Mrd. einführte; ein jüngeres Beispiel sind die Steuernachforderungen an die russischen Öloligarchen (an Chodorkovsky und seine Firma Yukos). Kydland und Pres$\operatorname{cott}^{32}$ waren die ersten, die auf die Zeitinkonsistenz öffentlicher Politikinterventionen auf Grund mangelnder Bindung hingewiesen haben (und dafür 2004 den Nobelpreis erhielten). Generell ist in einer Demokratie keine Regierung an die Versprechen der vorherigen

30 Dies verwendet die für sequenzielle Spiele typische Rückwärtsinduktion aus, d.h., zuerst wird die zweite Stufe, hier die Entscheidung des regulierten Unternehmens, und dann erst die erste Stufe analysisert.

31 So ist es optimal für Regierungen sich zu verpflichten, Forderungen von Terroristen nie nachzukommen. Jedoch in den konkreten Fällen wird dann dieses Versprechen oft gebrochen, etwa jüngst die italienische Regierung im Falle von Sgrena im Irak und Cantoni in Afghanistan.

32 Kydland/Prescott (1977). 
gebunden und bekannte Beispiele dafür sind die alternierenden Privatisierungen und Verstaatlichungen in Frankreich und in England und ein aktuelleres Beispiel ist das Aufweichen des Stabilitätspaktes nach dem deutschen Sündenfall.

In der Tat existiert kein Gleichgewicht (spieltheoretisch gesprochen) bei dem die Unternehmen in Kostensenkung investieren, wenn sich die Regulierungsbehörde nicht glaubwürdig zu einer Preisobergrenze verpflichten kann. Der Grund dafür ist, dass die Regulierungsbehörde ex-post nach Beobachtung der Kostensenkung immer den Anreiz hat, den Preis neu auf Basis der eben beobachteten niedrigeren Kosten festzulegen. Dazu kommt, dass eine politische Verpflichtung eher den Charakter eines Versprechens hat und somit Unsicherheit auf Grund der oben angesprochenen Gründe haften bleibt. In diesem Falle sind dann Misserfolge des regulierten Unternehmens bei der Kostensenkung eigentlich dem Regulierungsumfeld zuzuschreiben. Das bedeutet wiederum, dass den Vorteilen der öffentlichen Hand $^{33}$ bei der Bereitstellung von Anreizen gravierende Nachteile gegenüber Privaten gegenüberstehen, denn während sich Private zumindest auf vor Gericht einklagbare Verträge stützen können, mangelt es der öffentlichen Hand sich glaubwürdig zu binden. ${ }^{34}$

\section{Zusammenfassung}

Dieser Aufsatz versucht die Brücke zwischen Spieltheorie und Anwendungen in der öffentlichen Wirtschaft zu schlagen. Diese Ausführungen waren notwendigerweise auf einige Fälle - Strommärkte, Anreize, Regulierung - und einige grundlegende Konzepte beschränkt. Anstatt hier nochmals alles zu wiederholen seien hier einige potenziell für die Analyse interessante Themen vorgestellt. Dabei bieten sich zuallererst Auktionen an, die zunehmend zur Ausschreibung öffentlicher Leistungen (Baulose, Serviceverträge) und auch Titel (Frequenzen, Erdölfelder, etc.) verwendet werden und durchaus im Zusammenhang mit dem hier behandelten Aspekt privater Information stehen. Im Zusammenhang mit den Public Private Partnerships, die für zahlreiche Anwendungen ${ }^{35}$ denkbar sind, bietet sich die Theorie der Verfügungsrechte ${ }^{36}$ an, wobei einerseits ex-ante nichtkooperatives Nashverhalten und dann ex-post Kooperation (wiederum a la Nash) unterstellt wird. Dynamische Aspekte, auf die hier nur sehr indirekt im Regulierungsbeispiel eingegangen wurde, sind natürlich von eminenter Bedeutung für viele Bereiche der öffentlichen Wirtschaft, wo es um Investitionen geht. Mit zunehmender Privatisierung ist es auch von kartellrechtlicher Seite zunehmend notwendig zu überprüfen, ob hinreichende Konkurrenz (d.h. strategische Interaktion und nicht stillschweigende Kooperation) unter den Marktteilnehmern herrscht.

33 So sind die populären Public-Private-Partnerships, wo der private Partner vorwiegend für die Finanzierung zuständig ist, ein Unsinn, weil sich kein privater Partner zu den Zinskosten eines (entwickelten) Staates verschulden kann, vgl. Hart (2003).

34 Was der verstorbene österreichische Bundeskanzler Bruno Kreisky mit Floskeln „was kümmern mich meine Fehler von gestern“ abtat oder von vornherein die Klausel „rebus sic stantibus“ verwendete.

35 Ein Beispiel ist die Betreibung von Gefängnissen, vgl. Hart/Shleifer/Vishny (1997).

Hart (1995). 


\section{Anhang: Optimale Anreize an Hand eines Arbeitsvertrags}

Im Folgenden soll das Prinzip optimaler Anreize bei privater Information (inklusive der Arithmetik, allerdings ohne näher auf die Bedingungen einzugehen) an Hand eines Arbeitsvertrages vorgestellt werden und dabei auf die wichtigsten Konsequenzen hingewiesen werden. Die Arbeiter (= die Agenten) produzieren ein beobachtbaren Output $x$ abhängig (linear) vom nicht beobachtbaren Arbeitseinsatz $e$ und der individuellen Produktivität $t$, die private Information des jeweiligen Arbeiters ist:

$x=e \sqrt{ } t$.

Der Einsatz $e$ verursacht allen Arbeitern das gleiche (der Einfachheit wegen quadratische) Arbeitsleid, $K(e)=1 / 2 e^{2}$ so, dass die Produktionsvorschreibung $x(t)$ an den Typ $t$ folgende ,Auszahlung“ bedeutet:

$W(x, t) \equiv-K(e(x), t)=-1 / 2 x^{2} / t$.

Das (Brutto) Ziel des Prinzipals - hier der Umsatz - hängt vom Output der Agenten ab,

$V(x)=x$.

Da der Prinzipal den Typ $t$ (und auch den Einsatz e) nicht kennt, kann der (risikoneutrale) Prinzipal nur den erwarteten Gewinn durch die Wahl von Produktionsvorschreibungen $x(t))$ und kompensierenden Löhnen $(w(t))$ maximieren,

$$
\max _{\{x(t), w(t), t \in[\underline{t}, \bar{t}]\}} \quad \stackrel{\pi}{\pi=} \int_{\underline{t}}^{\bar{t}}[V(x(t))-w(t)] f(t) d t .
$$

Um diesen Erwartungswert zu berechnen, wird angenommen, dass der Prinzipal die zugrunde liegende Verteilung ( $F$ mit der Dichte $f$ ) der individuellen Arbeitsproduktivität $t$ kennt und das Intervall $[\underline{t}, \bar{t}]$ den gesamten Schwankungsbereich beschreibt. Zur Vervollständigung des Beispiels eines Arbeitsvertrages wählen wir dabei die Gleichverteilung:

$f(t)=\frac{1}{\bar{t}-\underline{t}}$ und $F(t)=\frac{t-\underline{t}}{\bar{t}-\underline{t}}$ für $t \in[\underline{t}, \bar{t}]$.

Eine bereits implizit verwendete Notwendigkeit für diesen Arbeitsvertrags (und auch allgemein) ist ein Transfer (= Lohn) an den Agenten (bezeichnet mit w), denn ohne diese finanzielle Kompensation $w$ würde der Arbeiter bei der Herstellung von $x$ nur verlieren (er würde mit seinem Arbeitsleid bezahlen) und daher den Vertrag nicht annehmen, oder 
schlicht und einfach nichts abliefern. Dies führt unmittelbar zu der ersten wichtigen Randbedingung eines jeden Vertrages, die der individuellen Rationalität,

$U=W+w \geq 0$,

d.h. der Gewinn des Agenten aus dem Vertrag $(U)$ darf nicht negativ sein, denn niemand wird einen solchen Vertrag freiwillig akzeptieren.

Es muss jedoch noch eine zweite Randbedingung berücksichtigt werden. Da der Prinzipal den Typ nicht kennt, kann und wird ein Agent vom Typ $t$ auf einen Vertrag jenen Typ $\hat{t}$ vorgegeben, der seinen Gewinn $\boldsymbol{U}(\hat{t}, t)$ maximiert,

$\max \boldsymbol{U}(\hat{t}, t) \equiv W(x(\hat{t}), t)+w(\hat{t})$.

$\hat{t}$

D.h., die Arbeiter wählen nicht notwendigerweise die für sie gedachten Aufgaben. Um diesen strategischen (und dadurch spieltheoretischen relevanten) Manipulationen durch die Agenten Rechnung zu tragen, müssen wir auf das wichtige Offenlegungsprinzip zurückgreifen: Ohne Verlust kann sich der Prinzipal auf Verträge $\{(x(t), w(t)), t \in[t, \bar{t}]\}$ beschränken, in denen es für den Agenten optimal ist, die Wahrheit zu sagen, also $\hat{t}=t$ zu bekennen.

Da auf Grund des Offenlegungsprinzip die Wahrheit zu berichten den Agentengewinn maximiert, reduziert sich der Agentengewinn auf eine Funktion von $t, U(t) \equiv \boldsymbol{U}(t, t)=$ $\max _{\hat{t}} U(\hat{t}, t)$, die wiederum folgender Bedingung für den Agentengewinn genügen muss (wegen des Enveloppentheorems):

$$
\frac{d U}{d t}=W_{t} \Rightarrow U(t)=\int_{t}^{t} W_{v}(x(v), v) d v
$$

Wenn $W_{t} \geq 0$ folgt, dass mit dem in (A8) mittels Integration berechneten $U(t)$ alle Agententypen $t$ einen nichtnegativen Gewinn aus dem Vertrag ziehen und daher akzeptieren. In anderen Worten, die Partizipationsbedingung (A6) bindet nur für den Typ $\underline{t}$, während für alle anderen Typen die Anreizkompatibilitätsbedingung (A8) bindet.

Substituierung von (A8) unter Verwendung von (A6) in das Zielfunktional des Prinzipals (A4) ergibt.

$$
\pi=\int_{t}^{t}\left[V(x(t))+W(x(t), t)-\int_{t}^{t} W_{v}(x(v), v) d v\right] f(t) d t
$$


Partielles Integrieren des Doppelintegrals in (A9) und Zusammenfassen liefert:

$$
\pi=\int_{t}^{t}\left[V(x(t))+W(x(t), t)-\frac{1-F(t)}{f(t)} W_{t}(x(t), t)\right] f(t) d t
$$

Punktweise Maximierung des Integranden in (A10) (durch differenzieren nach $x$ und anschließendes Nullsetzen) liefert folgende (notwendige) Bedingung für eine optimale Vorschreibung an Aktionen $x(t)$, das sogenannte relaxed program:

$$
V_{x}+W_{x}=\frac{1-F(t)}{f(t)} W_{x t}
$$

Beachte, dass die linke Seite gleich Null gesetzt, die effiziente oder erstbeste Lösung, $x_{1}(t)=t$ für Beispiel (A1) - (A3), ergibt und dass der Quotient auf der rechten Seite das Reziproke der Hassardrate $((\bar{t}-t)$ im Beispiel der Normalverteilung) ist. Da $F(\bar{t})=1$ folgt, dass der effizienteste Typ $\bar{t}$ die erstbeste Lösung erbringt, während alle anderen Typen unter der effizienten Lösung bleiben. Daraus folgt die interessante Implikation, dass Anreize auf der Ebene der Beobachtung die ohnedies gegebenen inhärenten Unterschiede der Typen noch verstärken. Diese Gleichung impliziert für den Arbeitsvertrag

$1-x / t=\left(x / t^{2}\right)(\bar{t}-t)=>x^{*}(t)=t^{2} / \bar{t}$.

Zuletzt sei noch die Bestimmung des Lohns, was zuerst die Berechnung des Agentennutzens $U$ erfordert, skizziert:

$$
\begin{aligned}
U(t)= & \int_{\underline{v}}^{t} W_{v}\left(x^{*}(v), v\right) d v=1 / 2 \int x^{* 2} / v^{2} d v=1 / 2 \int v^{2} / \bar{t}^{2} d v=v^{3} /\left.\left(6 \bar{t}^{2}\right)\right|_{\underline{t}} ^{t}=\left(t^{3}-\underline{t}^{3}\right) /\left(6 \bar{t}^{2}\right), \\
& \underline{t} \quad \underline{t} \\
w(t) & =U(t)-W\left(x^{*}(t), t\right)=\left(t^{3}-\underline{t}^{3}\right) /\left(6 \bar{t}^{2}\right)+1 / 2 t^{3} / \bar{t}^{2}=\left(4 t^{3}-\underline{t}^{3}\right) /\left(6 \bar{t}^{2}\right) .
\end{aligned}
$$

Diese Lösung ist in Abbildung A1 dargestellt, wobei zusätzlich das erstbeste Schema, eingezeichnet ist. Das optimale und gleichzeitig anreizkompatible Schema ist quadratisch und führt zu einer weit größeren Variation im Output und erfordert Lohnzahlungen, die den Arbeitern einen Nettonutzen lassen, wobei insbesondere die effizienten Typen Informationsrenten kassieren. Abbildung A1 zeigt das Ergebnis für ein Beispiel, $t \in[1,4]$; diese Variation im Typ bedeutet, dass der effizienteste Typ das doppelte bei gleichem Einsatz produziert. Im erst-besten Optimum ist diese Differenz auf den Faktor 4 gestiegen und im optimalen Arbeitsvertrag steigt dies auf das Achtfache, was unterstreicht, dass optimale Anreizverträge die individuellen Produktivitätsunterschiede verstärken. 

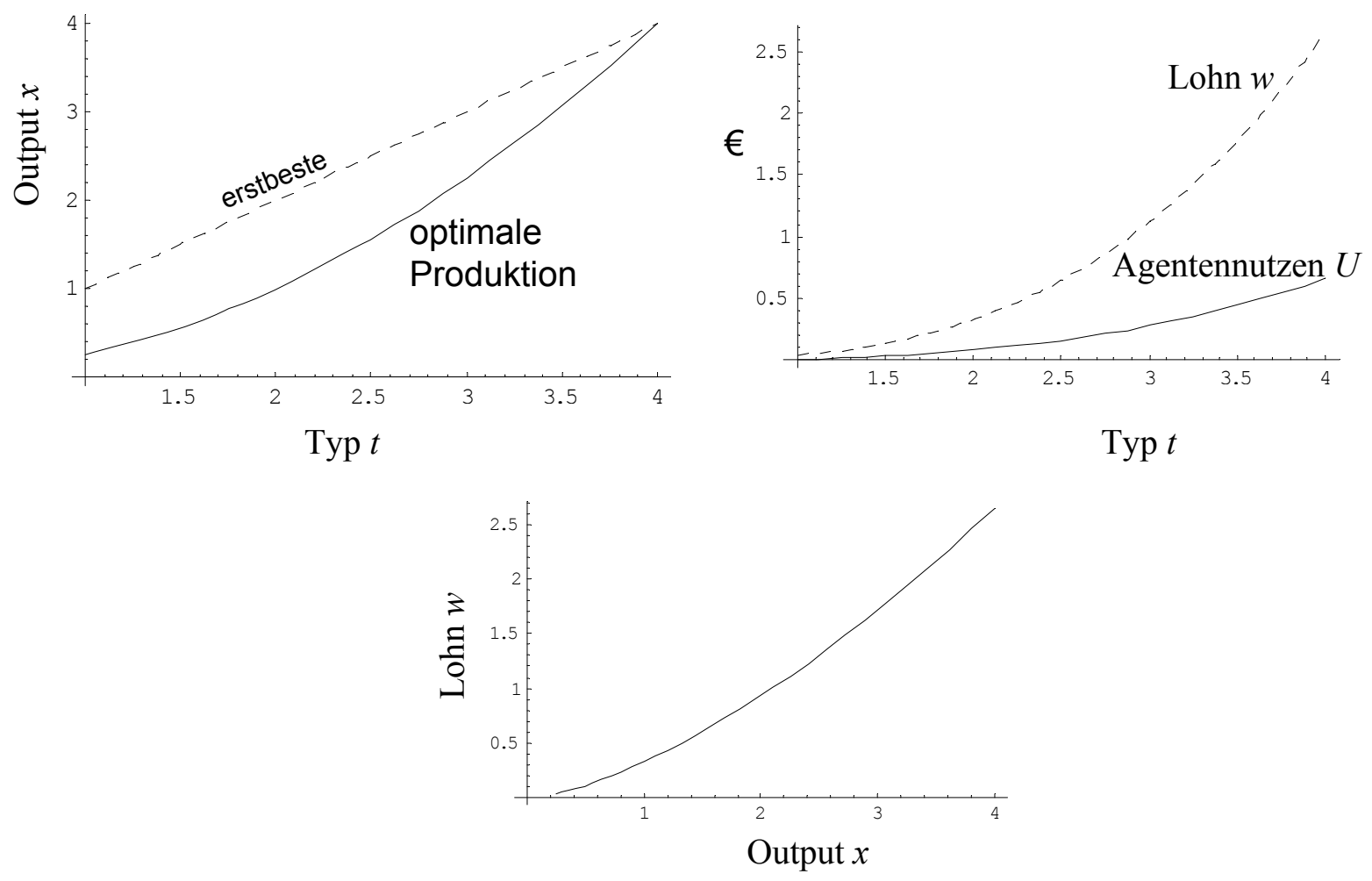

Abb. A1: Optimale Anreize: Produktionsziel (x), Nutzen (U) und Lohn (w) als Funktion des Typs $(t \in[1,4])$ und der Lohn als Funktion des Outputs.

Quelle: Eigene Darstellung

Dieses direkte Schema $\{x(\mathrm{t}), w(\mathrm{t}), t \in[\underline{t}, \bar{t}]\}$ scheint absurd, weil es explizit auf die private Information $t$ konditioniert ist, die dem Prinzipal ja fehlt! Diese Abhängigkeit ist jedoch ein rechentechnisches Artefakt, denn Eliminieren von $t$ liefert Lohn als Funktion des Outputs, also ein übliches Akkordlohnschema:

$w=\omega(x)=\left(4(x \bar{t})^{3 / 2}-\underline{t}^{3}\right) /\left(6 \bar{t}^{2}\right)$. 


\section{Abstract}

\section{Franz Wirl, A game-theoretical approach in the public sector}

Game theory; electricity pools; incentive compatibility; price caps

This paper presents applications of game theory to topical issues of public utilities and their regulation. Indeed, in some cases public utilities provide the best example for a game theoretic concept, e.g., electricity pools for competition in supply functions. In particular, this paper discusses different game theoretic concepts (matrix games, Bayesian mechanisms, sequential games, extensive versus normal form) and applies them to electricity exchanges, incentives and price regulation.

\section{Literaturverzeichnis}

Adachi, Mitsutishi M. (1999), On the Choice of Pricing Policies - ex ante Commitment and Prisoners ' Dilemma, in: European Economic Review, 43. Jg., S. 1647-1663

Allen, Victor (10. Juli 1996), A disability crutch, in: USA TODAY, S. 11A

Bolkestein, Frits (22. Mai 1999), The high road that leads out of the Low Counties, in: The Economist, S. 97-98

Bolle, Friedel (2001), Competition with supply and demand functions, in: Energy Economics, 23. Jg., S. 253-227

Dixit, Avinash K. und Susan Skeath (1999), Games of Strategy, New York und London

Dixit, Avinash K. und Barry J. Nalebuff (1993), Thinking Strategically - The Competitive Edge in Business, Politics, and Everyday Life, New-York und London

de Waal, Frans B.M. (April 2005), How Animals Do Business, in: Scientific American, S. 55

Fudenberg, Drew and Jean Tirole (1992), Game Theory, 2. Aufl., Cambridge (Mass.)

Green Richard J. and David M. Newbery (1992), Competition in the British Electricity Spot Market, in: Journal of Political Economy, 100. Jg., S. 992-953

Hart, Oliver (1995), Firms, Contracts and Financial Structure, Oxford

Hart, Oliver, Anrei Shleifer und Robert W. Vishny (1997), The proper scope of government - An application to prisons, in: Quarterly Journal of Economics, 112. Jg., S. 1127-1161

Klemperer, Paul D. und Margaret A. Meyer (1989), Supply Function Equilibria in Oligopoly under Uncertainty, in: Econometrica, 57. Jg., S. 1243-1277

Kreis, Constanze (2004), Deregulierung und Liberalisierung der europäischen Elektrizitätswirtschaft, Theoretische und empirische Befunde, Baden-Baden

Kydland, Finn und Edward C. Prescott (1977), Rules rather than Discretion - The Inconsistency of Optimal Plans, in: Journal of Political Economy, 85. Jg., S. 473-493

Laffont, Jean J. und Jean Tirole (1993), A Theory of Incentives in Procurement and Regulation, Cambridge (Mass.)

Leipzig Power Exchange (2002), http://www.lpx.de

Maynard Smith, John (1982), Evolution and the Theory of Games, Cambridge (Mass.)

McMillan James F., John Whalley und L. Zhu (1989), The Impact of China's Economic Reforms on Agricultural Productivity Growth, in: Journal of Political Economy, 97. Jg., S. 781 ff.

McMillan, John (1992), Games Strategies and Managers, Oxford

Newbery, David M. (2002), Problems of liberalising the electricity industry, in: European Economic Review, 46. Jg., S. 919- 927

o.V. (18. April 1999), The halt, the blind, the dyslexic, in: The Economist, o. Jg., S. 47

o.V. (22. Mai 1999), Down with disablement, in: The Economist, o. Jg., S. 18

Pankaj Ghemawat (1997), Games Businesses Play, Cases and Models, Cambridge (Mass.)

Rasmusen, Eric (2001), Games and Information - An Introduction to Game Theory, 3. Aufl., Oxford

Seidl, Christian (2001), Sind Unternehmer auch Menschen, in: Zum Erkenntnisstand der Betriebswirtschaftslehre am Beginn des 21. Jahrhunderts, Festschrift für Erich Loitlsberger zum 80. Geburtstag, hrsg. von Udo Wagner, S. 327-344, Berlin

Shleifer, Andrei (1985), A Theory of Yardstick Competition, in: Rand Journal of Economics, 16. Jg., S. 319-327

Wagner, Udo (Hrsg.) (2001), Zum Erkenntnisstand der Betriebswirtschaftslehre am Beginn des 21. Jahrhunderts, Berlin

Wirl, Franz (1997), The Economics of Conservation Programs, Boston, Dordrecht und London 
Wirl, Franz (1999), Conservation Incentives for Consumers, in: Journal of Regulatory Economics, 15. Jg., S. 23-40 Wirl, Franz, Claus Huber und Ian Walker (1998), Joint Implementation - Strategic Reactions and Possible Remedies, in: Environmental and Resource Economics, 12. Jg., S. 203-224 